\title{
E-SPORTS: DE LA PUERIL RECREACIÓN CON VIDEOJUEGOS A LA GAMIFICACIÓN DEL DEPORTE
}

\author{
E-SPORTS: FROM PUERILE RECREATION WITH VIDEO GAMES TO SPORT \\ GAMIFICATION
}

\begin{abstract}
Ramiro Buitrago Acuña ${ }^{1}$; Néstor Romero Ramos ${ }^{2}$; Omar El Kadi Janbeih ${ }^{3}$; Josué Vera Rodríguez ${ }^{4}$
\end{abstract}

\author{
${ }^{1}$ Abogado egresado de La Universidad del Zulia (LUZ), Abogado IV para la Alcaldía Boliviana de \\ Miranda, Estado Zulia, Venezuela. \\ dr.buitrago86@gmail.com \\ ORCID: 0000-0002-8563-7662; \\ ${ }^{2}$ Universidad Quality Leadership University, Ciudad de Panamá, Panamá. \\ nromero@qlu.ac.pa \\ ORCID:0000-0001-8147-2082 \\ ${ }^{3}$ Representante de fondos Mutuales y Consejero financiero para ScotiaBank, Canadá \\ oelkadi@urbe.edu.ve \\ ORCID: 0000-0002-5976-93X \\ ${ }^{4}$ Presidente Ejecutivo para Consultant and Partnership Alliance for Excellence, Inc., Puerto Rico. \\ jverarodriguez@pucpr.edu \\ ORCID: 0000-0002-0721-5069
}

Detalles del artículo:

Número de palabras: 7.315; Tablas: 0; Figuras: 0; Referencias: 23

Recibido: diciembre; Aceptado: diciembre; Publicado: enero 2020

Conflicto de interés: El autor declara que no existen conflictos de interés.

Correspondencia del autor: Ramiro Buitrago Acuña, dr.buitrago86@gmail.com

\section{RESUMEN}

El propósito del presente manuscrito comprende la materialización de un constructo teórico, crítico y dialéctico que aborde al gaming y el auge de los e-sports en la realidad sociocultural de la era moderna. Con un enfoque sofista; se da cuenta de las diferentes dimensiones que configuran la categoría abordada; auscultando los elementos necesarios, que con el tiempo elevaron la práctica con videojuegos hasta los desafíos del e-sports; donde no sólo se trata de recreación; también encuentran lugar la competición, el lucro y la dinámica deportiva. El artículo es del tipo documental, con diseño bibliográfico, en el cual se aplicó el método bibliográfico y la técnica de investigación documental, en el análisis e interpretación de los archivos seleccionados para tal fin. Tras una profunda discusión, se lograron resultados de interés para las ciencias: sociales, deportivas e incluso de la información; pues de forma razonable se describe el esfuerzo físico, neurológico y estratégico ejercido por los gamers, como miembros de equipos formalmente reconocidos por las comunidades aliadas a dicha industria. 
Finalmente, pudo concluirse que ante sus características y potencialidades; puede calificarse como disciplina deportiva a la práctica con videojuegos en línea (e-sports); donde múltiples videojugadores compiten por el éxito en la realidad virtual, lo que daría ocasión a la proyección de su perfil atlético profesional, enriquecimiento financiero y la fama a nivel mundial.

Palabras clave: e-sports, videojuegos, gamificación.

\section{Abstract}

The purpose of this manuscript includes the materialization of a theoretical, critical and dialectical construct that addresses gaming and the rise of e-sports in the socio-cultural reality of the modern era. With a sophisticated approach; realizes the different dimensions that make up the category addressed; auscultating the necessary elements, which eventually raised the practice with video games to the challenges of e-sports; where it's not just about recreation; competition, profit and sports dynamics are also found. The article is of the documentary type, with bibliographic design, in which the bibliographic method and the documentary research technique were applied, in the analysis and interpretation of the files selected for this purpose. After an in-depth discussion, results of interest to the sciences were achieved: social, sports and even information; because the physical, neurological and strategic effort exerted by the gamers is reasonably described as team members formally recognized by the communities allied to that industry. Finally, it could be concluded that given its characteristics and potentialities; it can be qualified as a sport discipline to practice with online video games (e-sports); where multiple video players compete for success in virtual reality, which would give rise to the projection of their professional athletic profile, financial enrichment and worldwide fame.

Key words: e-sports, video games, gamification.

\section{INTRODUCCION}

Con el acaecimiento de numerosos eventos sociales vinculados a la cultura digital y el extravagante, rutilante y casi omnipresente desarrollo tecnológico, han surgido diversas manifestaciones del comportamiento humano, de las que no se daría cuenta hasta los últimos 10 años, lo cual les reviste de cierta aura novedosa, para los estudiosos del pensamiento humano o quienes se han decantado por el desarrollo de los estudios del hombre. Para nadie es un secreto que con el uso de herramientas tecnológicas, se facilita el desarrollo de acciones/procesos comunes y complejos en casi cualquier escenario: humano, social, deportivo, médico, económico, político, cultural e incluso bélico.

Conforme a lo anterior, es más que natural surjan diversos interesados por comprender la naturaleza de los procesos humanos, donde hasta entonces ha intervenido la tecnología: comunicación, empoderamiento, economía, deporte, liderazgo... por mencionar algunos. Todos estos eventos, tienen en común que tras su desarrollo, potencialización y transformación han estado presentes las ciencias aplicadas. Por ello, emerge esa curiosidad mórbida por concretar y definir el alcance logrado hasta entonces, por la tecnología en tales escenarios, interviniendo aún incluso en la realidad humana más pura: el deporte.

Con referencia a lo descrito en el párrafo de arriba, los autores se avocaron por definir hasta donde la tecnología ha tejido los hilos de la humanidad, sobre todo en la última década... 
a tal punto que ciertamente logró desestructurar y deconstruir los esquemas clásicos que enmarcaron las dinámicas humanas durante más de 300 años; entre las que se cuentan: la pareja, el sexo, la familia, trabajo, recreación, ocio y el deporte. Es decir, que todos estos procesos humanos neurálgicos, fundamentales, seculares, se desarrollan hoy con apoyo en las herramientas tecnológicas, de modo recurrente y algunas veces absoluto, cuestión de la cual la sociedad "moderna" se ufana, sin darse por aludida en que con ello, su humanidad adquiere cierta plasticidad informática.

En ese sentido, las diferentes herramientas tecnológicas se han convertido en extensiones corpóreas de los hombres, que hacen uso y aprovechamiento de estas; claro está, unas más que otras, tal es el caso de los teléfonos inteligentes, tabletas, Ipad, Ipod, PSP, entre otros dispositivos móviles, como los mandos de las diferentes formas de consólas que existen en el mercado y con las que los usuarios se recrean y juegan con videojuegos. Estas últimas llaman la atención, por su inminente presencia en los hogares de hombres adultos, entre los 25 y 45 años de edad. Lo cual lleva a preguntarse: ¿Qué acaso los videojuegos no suelen ser factor de divertimento infantojuvenil?

Por ello, en el desarrollo del presente extenso, se han propuesto los autores... profundizar sobre los videojuegos, aunque más allá de abordarles como fenómeno tecnológico, se persigue dilucidar la simbología que estos han alcanzado en el espectro deportivo, social y cultural; así como también el origen de otros eventos, como respuesta al auge de los mismos y su potencialización con apoyo a las distintas plataformas, que en la realidad virtual generan espacios a la socialización, recreación, competición y monetización del uso de videojuegos, no tanto por el tiempo que los gamers se dediquen a ello, sino al alcance de su proyección como acontecimiento de interés internacional: e-sports. En lo que a esto respecta Roncero (2019), explica:

El tratamiento mediático que, en ocasiones, reciben los videojuegos es un reflejo de esta visión asociada del juego como un acto infantil. Lo mismo ocurre con el deporte electrónico, una actividad que ha sido asociada por parte de la sociedad como un fenómeno pueril.

Ahora bien, tras los planteamientos anteriores, es válido rescatar todo cuanto ocurre en la sociedad moderna, globalizada, no sólo es con ocasión al adelanto tecnológico existente, es también muy cierto que el hombre, desde su origen, por aquellos primeros días que comenzara a interactuar y socializar, se sintió interesado por la recreación y el juego. La distracción y enriquecimiento lúdico, como terapia autodidacta en el desarrollo consciente e inconsciente de habilidades y destrezas neuronales, pues en su desarrollo personal, los seres humanos se muestran complejos, egoístas, caprichosos, agresivos, generosos, gregarios, emocionales y racionales, cuestión que es parte de su propia naturaleza. Al respecto Hernández y Buitrago (2017), afirman que:

El individuo en sí es un mar complejo donde se funden indistintos organismos que permiten la creación de una efigie personal. Elementos heredados como la constitución corporal, o algunos adquiridos posteriormente, como el temperamento o el carácter, constituyen la esencia básica del ser homínido.

Así pues, resulta cardinal reconocer que lo mismo ha influido en el interés preponderante y abismal generado por los videojuegos, cuyo uso se ha potencializado con el 
aprovechamiento del internet; sobre todo en los países del primer mundo, donde jugarlos en tiempo real, compitiendo con otros gamers, es algo más que simple recreación, ha alcanzado su propia denominación: e-sports; todo lo cual ha dado lugar al desarrollo de una industria pluridimensional, cuyo espectro cubre la invención de videojuegos, su producción, mercadeo, comercialización, innovación, desarrollo de cultura especifica e incluso ha brindado oportunidad al desarrollo de una carrera a los videojugadores, quienes pudieran alcanzar un estatus superior, con reconocimiento institucional e internacional a destacadas figuras del gaming: cyberathletes.

Conforme a lo anterior Roncero (2014), afirma que: "El proceso de convergencia mediática y la democratización informativa, potencializó al sector de los videojuegos, elevandoles a los deportes electrónicos, un nivel jamás visto, tanto en popularidad mediática, como en rentabilidad económica e impacto publicitario". Por su parte Salcedo (2014), plantea: "Los videojuegos dejan de ser un predictor de consecuencias negativas, para convertirse en un factor que podría colaborar en los procesos de desarrollo de algunas capacidades tanto cognitivas como funcionales".

Entre tanto Aranda, Sánchez y Martínez (2016), opinan que: "El juego digital goza de una presencia plenamente consolidada en el ecosistema mediático actual. Muestra de ello es su creciente diversificación y especialización, tanto en temas y géneros como en plataformas de distribución". Por esto, se toma en cuenta a Roncero (2019), quien explica: "el deporte electrónico, como fenómeno, difiere del videojuego, como medio, en los rasgos particulares que lo conforman como un elemento diferenciado del medio original, aunque no puede desligarse de los rasgos generales de los que nace".

En opinión de autores como kopp (2017), quien afirma que: "los deportes electrónicos corresponden a una forma de participación en los videojuegos, que deja de lado el aspecto meramente lúdico y de diversión; centrandose en hacer del juego una profesión, tomando características de competición, entrenamiento y rendimiento". Claramente el citado autor expone que son los e-sports, dando una suscinta pero efectiva explicación del evento abordado. Por su parte Chiva, Pallarès e Isidori (2017), plantean que:

El mundo vertiginoso en el que vivimos, en pleno siglo XXI, contribuye y condiciona la evolución del deporte. Tanto es así que últimamente estamos asistiendo a un fenómeno que nos obliga a replantearnos algunas de las concepciones que teníamos sobre el deporte: seis jóvenes uniformados salen a escena en medio de un arco iris de luces y de música y se sientan a escasos centímetros de un teclado mientras veinte mil personas aplauden desde las gradas de un estadio.

Por ello, se toma en cuenta a Roncero (2019), quien explica: "El juego, en general, y los videojuegos, en particular, han estado tradicionalmente identificados como una actividad propia de un público infantil e impropia del público adulto". Prosigue el mismo Roncero (2014), el cual afirma: "La vertiente competitiva ha desarrollado un complejo proceso de profesionalización que está contribuyendo a un cambio en la percepción que la sociedad tiene de ellos".

En la opinión de García (2019), sucede que: "Los e-sports son una actividad humana de rendimiento (se considere técnicamente o no como deporte), con un alto contenido mental 
(como, por ejemplo, el ajedrez), un ámbito de actuación y una oportunidad laboral para el psicólogo del deporte". En ese mismo orden de ideas, autores como Beltrán, Valencia y Molina (2011), afirman: "En una sociedad inmersa en las tecnologías y con elevados índices de sedentarismo, los videojuegos activos representan un nuevo fenómeno social que puede comportar beneficios para la salud pública”. Por su parte kopp (2017), plantea:

Jugar profesionalmente conlleva una forma diferente de hacerlo, orientada a la eficiencia, la participación recurrente en torneos, competencias, la transmisión de sus partidas y resultados a través de redes sociales como prácticas habituales. Esto trae aparejado que el juego, como deporte electrónico sea una hibridación entre ocio, deporte y trabajo, fusionando diversión y responsabilidad.

Desde su basto conocimiento, los expertos Beltrán, Valencia y Molina (2011), plantea que: "Los videojuegos activos permiten la interacción física de los jugadores y sus movimientos con la realidad virtual, que aparece en pantalla a través de diferentes dispositivos". Lo anterior, genera expectativas con relación al movimiento, agilidad, destreza, equilibrio y dominio corporal por parte de cada gamer, lo cual es un atributo significativo del videojuego activo. Por su parte Gutierro (2019), expone: "Frente a la visión analítico-estructural se abrió paso, en la segunda mitad del siglo XX, una comprensión holística de la vida".

Entre tanto Roncero (2019), afirma que: "Con los últimos acontecimientos, se abre una gran puerta al estudio de los videojuegos, como producto cultural atendiendo a diferentes aspectos, relacionados con los cambios socioculturales, producidos por la interacción entre el mundo virtual y real". De igual forma, el mismo y antes citado García (2019), explica que: "Psicología del Deporte se encuentra en un período de integración de estrategias psicológicas de intervención y ampliación de ámbitos de actuación más que de exclusión, como sucede en los esports".

En ese sentido, los autores del presente manuscrito, proponen desarrollar un discurso sofista orientado a redescubrir y descubrir: ¿De que forma la tecnología ha transformado los procesos recreativos vinculados al deporte?. Para dar respuesta a esta y otras interrogantes inherentes al mismo evento: e-sports; resulta fundamental profundizar en ello.- ¿Qué es?, ¿En que consiste?, ¿Quiénes intervienen?, ¿Cuál es su propósito?, ¿Cuándo encontraron su origen?; respecto a lo anterior, emerge una categoría de investigación transdisciplinaria, donde el deporte, la ingeniería, sociología e incluso antropología dan cuenta del comportamiento manifestado por los cyberathletes en la era digital.

\section{MATERIAL Y MÉTODOS}

\section{Diseño}

El artículo en cuestión es del tipo documental; en su elaboración se aplicó el método bibliográfico, donde se aprovechó el diseño bibliográfico con enfoque crítico y dialéctico, para su perfeccionamiento se utilizó como técnica la documentación, al considerarle como la más indicada, ante la naturaleza del contenido abordado. El manuscrito, presenta en su extensión el acervo doctrinal reunido por los autores, al tiempo que hace referencia a distintos hallazgos alcanzados por investigaciones previas, que en el pasado abordaron dicha temática; a lo anterior 
se adicionan los laudos sumariales, propuestos por quienes realizaron el estudio, fundamentándose en la información escrita con la que se documentaran para su investigación.

Con atención a lo descrito en el párrafo anterior, se esgrime al presente manuscrito como documental; en ello se desarrolló el análisis e interpretación crítica y dialéctica de documentos escritos, en los cuales reposaba el contenido informativo y epistemológico con origen en investigaciones previas, que más allá de ser antecedente al presente texto, fungieron como faros de luz en la búsqueda del conocimiento nuevo, el descubrimiento y redescubrimiento de dimensiones diferentes o similares a las ya abordadas por otros investigadores. Al respecto Arias (2012), indica que la investigación documental, es un proceso basado en la búsqueda, recuperación, análisis, crítica e interpretación de datos secundarios, es decir, los obtenidos y registrados por otros investigadores en fuentes documentales, impresas, audiovisuales o electrónicas.

\section{Documentos revisados}

Para la elaboración del presente escrito, se seleccionaron diferentes publicaciones científicas y académicas, generadas por expertos en las ciencias sociales, de la ingeniería y el deporte, artículos arbitrados en su mayoría. Tales documentos fueron auscultados desde el análisis crítico e interpretativo. Al respecto, Sabino (2006), plantea que las investigaciones de corte descriptivo, se proponen conocer grupos homogéneos de fenómenos, para poder establecer así una descripción única de sus características y el ambiente. En ese sentido, el extenso en cuestión, presenta los hallazgos finales, logrados por los autores, al concluir el proceso indagativo.

Los escritos seleccionados conforman una serie de documentos recogidos por los autores, las cuales ascendieron a la cantidad de 50 diferentes títulos; tras un procedimiento discriminativo, selectivo y sistematizado, los cuales... integran un cúmulo de archivos documentales de interés social, cultural, deportivo, económico e incluso tecnológico y que finalmente serían reducidos a 23, referidos al final del extenso. Estos, fueron intervenidos con la aplicación de métodos/técnicas documentales y bibliográficas, las que serán descritas y explicadas en lo sucesivo y que permitieron generar este cuerpo documental; donde se concretarían elementos de naturaleza universal, dando origen a una categoría investigativa novedosa para las ciencias del deporte: e-sports.

Conforme a lo anterior, los autores seleccionaron la técnica de investigación documental, dado su curso de acción, así como también la naturaleza del trabajo indagativo; al aplicar la técnica de documentación, se originan contenidos de tipo cualitativo, pues en los procedimientos investigativos donde es aprovechada, generalmente o casi siempre.... se ofrecen hallazgos de interés neurálgico, lo cual funge como soporte doctrinal y epistémico a la indagación que se esté llevando a cabo. Lechuga, Leyva y Cazallo (2018), afirman: "En este tipo de técnica se utiliza como instrumento de investigación: las ideas, argumentos y proyectos que fueron interpretados desde una perspectiva analítica y crítica".

Así entonces, se trata de un artículo documental, donde se presentan sumarios descriptivos, en el que con un enfoque sofista, los autores dan cuenta del impacto del gaming en las dinámicas deportivas profesionales con apoyo a las tecnologías de la información y comunicación a las que en adelante se hará mención como TIC y que dieron oportunidad al 
surgimiento de los e-sports, temática sobre la que versa el presente extenso. Para el experto en este tipo de investigaciones Tamayo y Tamayo (2007), estudios de tal naturaleza presentan la descripción, registro, análisis e interpretación del contenido de procesos o eventos, al tiempo que expone tras su auscultación, la realidad de hechos vinculados al origen del objeto de estudio, interpretandoles de manera correcta.

Para Sierra (2010), dichos estudios conforman el análisis de informaciones que reposan en documentos, los cuales al ser organizados de forma correcta, dan origen al nuevo conocimiento, teniendo en cuenta una previa discriminación de datos e información que realmente favorezcan y enriquezcan el estudio en cuestión. Al respecto, Sabino (2006), concibe que las investigaciones descriptivas, aspiran conocer grupos homogéneos de fenómenos para con ello lograr una descripción concreta de sus características propias. En opinión de los comunmente citados Hernández, Fernández \& Baptista (2006), la investigación sobre la que versa este párrafo; busca especificar las propiedades importantes de las personas, grupos, comunidades o cualquier otro evento que sea sometido a análisis.

Con la elaboración del manuscrito, fue necesario el desarrollo de la búsqueda preliminar de archivos, que presentasen los datos e información necesaria y pertinente, favoreciendo la concertación de un extenso cuyo contenido resultase interesante al lector, al punto que con el, se inspirase el inicio de otras tantas investigaciones con abordaje sobre la misma temática: esports. Es menester destacar que cada una de las fuentes auscultadas, fue de naturaleza bibliográfica y documental, pero por sobre todo escritas, de tipo físico y digital, las que finalmente fueron referidas al final del extenso. El diseño de esta investigación, consistió en la revisión bibliografíca de documentos, contentivos de los hallazgos socializados por publicaciones científicas: revistas arbitradas de habla hispana en países del continente americano y europeo.

Igualmente, al inicio del procedimiento científico, además de consultar diversas obras de la literatura académica; se tomaron en cuenta repositorios digitales de revistas científicas y arbitradas; adscritas a grandes y reconocidas redes de intercambio del conocimiento, como: Latindex, Redalyc, Publindex, Colciencias... entre otras; lo cual respalda la alta calidad editorial de los contenidos aprovechados por los autores, desde la definición del acceso abierto. Para lograr identificar en estas plataformas digitales, tales o cuales serían los archivos de apoyo al desarrollo del presente estudio, se utilizaron como palabras de búsqueda: e-Sports, videogames, gaming, gamer, cyberathletes, gamification.

Del mismo modo, los criterios de inclusión/exclusión de la bibliografía rescatada de dichas bases de datos, se fundamentaron en su carácter cultural, simbólico, innovador, holístico, humano y social; es decir que todos y cada uno de los artículos citados y referidos al final del escrito, están allí porque por sí solos representan contenido de interés para las ciencias sociales y humanas... entre las que se cuentan a las del deporte; así como para las de la ingeniería, por cuanto todos los aspectos que rodean al videojuego, revisten gran importancia para su desarrollo como producto. Así entonces, logró condensarse en un sólo cuerpo escrito, las teorías de los expertos y los laudos epistémicos de los autores, acerca de una temática que tiene mucho que ofrecer como categoría científica: e-Sports. 


\section{RESULTADOS}

\section{E-Sports: ¿Profesionalización atlética por dedicación o lucro?}

Al haber identificado cuales son algunos de los elementos que integran esta nueva forma de competir en la era moderna, mitad virtual, mitad real, donde quienes se sientan identificados con este tipo de dinámicas que comprenden diferentes dimensiones como: liderazgo, competición, empoderamiento, estrategia, confrontación, conformación de equipos, camaradería, emoción, agilidad neuronal y física (motricidad fina y destreza ocular/visual), pudiera comprenderse el por qué ha llegado a considerarse como proceso deportivo; pues todo lo mencionado en este párrafo también calaria al hablar de disciplinas deportivas clásicas a saber: waterpolo, polo, beisbol, futbol, voleibol, softball, golf... entre otros.

Comprendido lo anterior, y con una visión mucho mas amplia sobre las dinámicas humanas y la forma en que estas se desarrollan con lugar en la era digital, podrían identificarse incluso otros elementos afines entres los e-sports y los demás deportes. Desde su experiencia Sánchez, Ramirez, Baez, Moreno y Fernández (2019), explican: “Actualmente es difícil imaginarnos nuestro día a día sin las Nuevas Tecnologías, sin todos aquellos aparatos que sirven para buscar e intercambiar información o para que las personas se comuniquen entre sí. Son utilizadas en todas partes del mundo, y cada día más gente se une a su uso".

Ahora bien, usualmente las palabras significan lo que en verdad significan, asi también ocurre con las palabras compuestas y eletronic - sports no es la excepción. Para Roncero (2014), sucede que: "Los deportes electrónicos o e-sports son el resultado de la profesionalización del mundo competitivo de los videojuegos, un fenómeno reciente que se ha desarrollado con fuerza en los últimos años". En ese sentido y según la visión de García (2019), quien expresa:

Se puede decir que existen más similitudes que diferencias entre los atletas de los $e$ sports y los deportes tradicionales al realizar un trabajo psicológico. Al fin y al cabo, se trabaja con personas (gamer/ entrenador) que tienen sus propias necesidades psicológicas a cubrir como cualquier otro deportista (p.ej., motivación, concentración, control del estrés, confianza, etc.). Lo que varía es la actividad (demanda y rendimiento en un videojuego), el perfil del gamer/ entrenador (p.ej., en algunos casos, sufren estrés competitivo, fatiga mental, baja tolerancia a la frustración, falta de esfuerzo y trabajo, etc.) y la interacción bidireccional que se da entre ambos (persona-videojuego).

Con referencia a lo anterior, es necesario explicar el origen psicologíco de donde emerge el interés por profesionalizarse en el ambiente gaming. En opinión de autores como kopp (2017), quien afirma que: "Profesionalizarse en el gaming parece estar unido a la necesidad de mostrarse o hacerse conocido, es decir, formar parte tanto de la competición como de la espectacularización del juego. Por esta razón, los jugadores dedican parte de su tiempo a transmitir sus sesiones de juego". En ese orden de ideas el mismo Keopp (2017), plantea:

Esta difusión se da mayoritariamente a través de dos canales: las plataformas Twitch y Youtube. (...), el sitio Twitch permite transmitir en vivo, tanto el video capturado por una cámara web, como las acciones en pantalla, permitiendo al jugador mostrarse y aquello que está jugando en tiempo real, además de comunicarse vía chat con su audiencia. Youtube es una 
plataforma de videos, en la cual los jugadores también postean fragmentos relevantes de sus partidas o bien, fragmentos de los streamings realizados en Twitch. Asimismo, a través de los comentarios de los espectadores en las redes sociales y en el chat que acompañan la transmisión, los jugadores generan una interacción que los sitúa en un lugar similar a performers o personajes de entretenimiento.

Bajo el orden de las ideas anteriores, puede observarse como las TIC con apoyo en la globalización, internacionalización y masificación en la práctica con videojuegos y por supuesto el auge del internet, han transformado incluso la idea del atleta, el hombre moderno que se recrea, deconstruye y robustece con la práctica deportiva... aún en una realidad alterna o dual. Para los expertos en ciencias del comportamiento humano, García y Santizo (2010), sucede que: "En el mundo del siglo XXI, la era de la globalización, las comunicaciones y la información, forman parte del día a día de cada persona". En opinión de autores como kopp (2017), quien plantea que:

Tal como sucede en la actualidad con deportes como el fútbol o el básquet, la profesionalización de la actividad; está acompañada tanto de una espectacularización como de una monetarización. Este último aspecto, va más allá de la búsqueda de los jugadores de obtener réditos; sino que se trata de un complejo entramado de prácticas e intereses puestos en juego, tanto por empresas vinculadas al mundo del entretenimiento, la tecnología y los videojuegos. Los principales rubros auspiciantes de las competencias en deportes electrónicos, son empresas de Hardware informático (Intel, AMD, entre otras), portales de noticias y comunidades especializadas en videojuegos (IGN, Gamestop), plataformas de streaming (Youtube y Twitch) y marcas de bebidas energizantes (Coca-Cola, Red-Bull).

Por otro lado, no es fácil que quienes comúnmente manifiesten estar interesados en el mundo del deporte, conciban a los e-sports como otra disciplina de la misma naturaleza, tomando en cuenta que para poder intervenir en ello, no es necesario ser tan atlético o ajustarse a un perfil físico determinado, como en el caso del boxing por mencionar alguno. Así entonces cabe preguntarse... ¿Acaso no ocurre del mismo modo con quienes practican el ajedrez de forma profesional?. En opinión de García (2019), sucede que: "La gente que venimos del deporte tradicional, de alguna forma, somos "intrusos" o "nuevos en el sector", por lo que se requiere tiempo, trabajo y convencimiento. La gente de los e-sports, han vivido siempre la misma realidad y es lo que ellos conocen".

Por ello, en este artículo se plantea que para atender todos estos vacíos, es fundamental que diferentes disciplinas de las ciencias del deporte, sociales y humanas, profundicen en el fenómeno moderno del videojuego y todo lo que este significa, desde la dimensión cultural, deportiva, psicológica e incluso económica. Por su parte García (2019), expresa: "La Psicología del Deporte tiene grandes retos a alcanzar en los e-sports como es la regulación y formación de los psicólogos que quieran trabajar en este contexto". Respecto a ello, llama la atención aquello propuesto por los autores Santos, Alloza y Escribano (2018), quienes afirman: "Algo a lo que llamamos internamente Gamificación Inversa. En ello, partimos de la hipótesis en la que cualquier videojuego puede entrenar ciertas habilidades, entre ellas las llamadas soft skills".

Finalmente, puede apreciarse cómo con la integración de fenómenos de distinta naturaleza, entre los que se cuentan: la globalización, el internet, multiculturalidad, tele presencia, videojuegos, gamer, TIC, internacionalización de marcas, el intercambio 
comercial, entre otros; surgen nuevos eventos como es el caso de los e-sports, abordados en este extenso como categoría de interés científico social, humano y deportivo. En ello, se encumbra el propósito acordado por los autores del presente artículo... de cara al auge de las nuevas tecnologías con la informatización de las prácticas deportivas.

\section{DISCUSIÓN}

Indistintamente de los intereses que puedan exponerse con relación a los videojuegos o los video jugadores, es importante reconocer que todo este fenómeno ostenta un trasfondo cultural, social, humano, como aquella necesidad que tienen los seres humanos, en especial los del sexo masculino, en proyectarse como el mejor, el más fuerte, el más sabio, el más ágil, el vencedor, el ganador... de ahí que en su mayoría los gamers resulten ser hombres. Y es que ciertamente, de allí emerge el mismo interés por llevarlo al nivel competitivo e identificarse entre sus pares, distinguiéndose como quien mejor juega, el más ágil o más estratega al momento de "jugar". Al respecto, Roncero (2019), plantea que:

A pesar de que los deportes electrónicos es un fenómeno derivado de los videojuegos, van un paso más allá que el propio medio. Así lo han establecido varios autores que han observado las diferencias conceptuales entre videojuegos y deportes electrónicos, identificando el segundo concepto como una evolución y modificación de la idea tradicional de videojuego.

De allí, toda esta masificación con el uso de videojuegos, el surgimientos del gaming o aún más allá de ello, el gamer... ¿Dónde tuvo origen todo este evento?. Lo más elemental que se debe recordar, es que como se dijo al inicio del extenso, los seres humanos tienden a procurar su entretenimiento, recreación o la simple satisfacción del interés por sentir que está haciendo algo, como aquella idea del ocio productivo... y ¿qué mejor que llenar tal vacío con experiencias estéticamente coloridas?, donde pueden los hombres generar un alter ego, que todo lo puede, con habilidades y destrezas metahumanas, en una realidad fantasiosa, que a pesar de ser virtual pueda compartir en tiempo real con otros hombres y tener la sensación real de intervenir estos escenarios, claro está con aprovechamiento de las TIC, ejerciendo su tele presencia. Al respecto Aranda, Sánchez y Martínez (2016), plantean que:

Nuestra relación con los productos de la cultura popular, funciona a través de la producción de estructuras de placer, y el juego digital no es una excepción. Analizar de qué modos se crean estas estructuras de placer, es fundamental para entender el peso cultural de un fenómeno como el de los videojuegos.

Igualmente, es necesario recordar donde tuvo oportunidad de cocrearse tal evento, pues para tener una idea de hasta donde se puede llegar o incluso que alcance se aspira lograr en la práctica con videojuegos a nivel profesional: e-sports Resulta provechoso saber cuándo y cómo es que todo comenzó. Respecto a ello, en un informe donde recogen parte de la historia de los deportes electrónicos, Fundación Telefónica (2017), relata que:

El origen de los e-Sports actuales hay que buscarlo hace más de 40 años en la Universidad de Stanford, donde tuvo lugar en 1972 el primer torneo de videojuegos entre estudiantes con el juego Spacewar. En 1980, con el popular Space Invaders, uno de los videojuegos comerciales pioneros, la empresa Atari atrajo a más de 10.000 concursantes de todo Estados Unidos. Otra de las primeras competiciones de e-sports fue Red Annihilation, en 
1997. El cambio de siglo vio nacer las primeras ligas de e-sports: Electronic Sports League y Korea e-sports Association (KeSPA). En 2006 entró en escena Justin.TV, una plataforma de video streaming que cinco años más tarde dio lugar a Twitch.TV, dedicada en exclusiva a las partidas de videojuegos. 2009 es el año de lanzamiento al mercado de League of Legends, el juego de estrategia que cuenta con más usuarios en todo el mundo. Tres años más tarde, en 2012, la cadena de televisión coreana OGN (Ongamenet) comenzó a retransmitir sus campeonatos en directo. Y este breve esbozo cronológico se cierra con el hito que marcó en 2016 el campeonato mundial del League of Legends, cuando fue visto por 43 millones de usuarios.

Expertos como en el caso de Checa (2009), afirman que: "En la sociedad española, como en general en toda la sociedad occidental, se ha hecho evidente en los últimos años un cambio cualitativo relevante en torno a los videojuegos, que alcanza a los propios gobiernos. Los videojuegos tienden a ser contemplados como una creación cultural de enorme potencialidad". Por su parte Chiavenato (2009), explica que:

El conocimiento humano se está convirtiendo en el principal factor de producción de riqueza. Los factores tradicionales de la producción (tierra, capital y trabajo) se han agotado y su utilización ha dejado de contribuir a la eficiencia y la eficacia. Fueron excelentes motores en la era industrial, pero en la era del conocimiento; el mundo de los negocios depende cada vez más de la producción de ideas y conceptos, y los servicios y la información que se derivan del conocimiento, se están convirtiendo en bienes esenciales que se intercambian en el mercado. Si bien continuamos vendiendo y comprando bienes materiales, las mercancías son creadas y producidas por ideas que provienen de procesos de investigación y desarrollo. El saber no es un bien económico en el sentido clásico, porque su uso no lo destruye y su transferencia no representa una pérdida para quien lo posee. Al contrario de los bienes materiales, sujetos a la depreciación y la descomposición, cuanto más se utiliza, difunde y divide el conocimiento, más valor adquiere.

Del mismo modo, el ya referido Checa (2009), opina que: "En la sociedad española y europea, se ha producido un cambio substancial en la apreciación de los videojuegos, pasaron de ser una afición juvenil que llenara horas de ocio, a un modelo de comunicación audiovisual con múltiples aplicaciones positivas". En la opinión de García (2019), sucede que:

Existen algunos retos a conquistar dentro de los clubes y equipos de e-sports, pues se requiere desarrollar culturas de alto rendimiento desde una perspectiva interdisciplinar, rescatar lo positivo de los e-sports e integrar cuestiones contrastadas de los deportes tradicionales. Puede que, en los e-sports, como en cualquier otro contexto, haya una cierta resistencia al cambio, aunque al mismo tiempo, una positiva apertura a la mejora e innovación.

Por su parte Checa (2009), opina que: "En los años iniciales del nuevo siglo, la influencia de los videojuegos ha sido casi obsesivamente analizada a través de estudios, más o menos ambiciosos y científicos, desarrollados en buena medida en el seno de las universidades españolas". Entre tanto Roncero (2019), plantea que: "Si atendemos de manera concreta al estudio de los deportes electrónicos, podemos observar la superficialidad con la que se ha tratado el fenómeno desde el campo de las ciencias sociales". Los autores Santos, Alloza y Escribano (2018), afirman que: 
Entre las habilidades más implicadas en los e-sports se cuentan: la velocidad de Razonamiento; una habilidad cognitiva que se puede definir como el tiempo que le lleva a una persona hacer una tarea mental, implica la capacidad de realizar con fluidez las tareas fáciles o ya aprendidas. Al mismo tiempo genera se desarrolla una gran capacidad para tolerar el fallo continuo una y otra vez, tratando de lograr un objetivo, así también favorece la gestión del Estrés. Ser capaz de mantener el rendimiento mientras te estresan ciertos estímulos.

Al socializar las ideas de Roncero (2014), sucede que: "Los deportes digitales se plantean como un mercado en potencia con grandes posibilidades durante los próximos años. En esta línea, se echa en falta una apuesta firme por parte de los grandes medios de comunicación para explorar su potencialidad". Por su parte, expertos de la talla de Fanjul, González y Peña (2019), expresan:

El patrocinio de un equipo en los deportes electrónicos, es una estrategia eficiente que permite desarrollar acciones de contenido de marca (branded content) vinculadas a los mismos. La mera presencia de la marca en las competiciones, también genera reconocimiento mediante el componente de aspiración, y una perfecta manera es creando un equipo de jugadores profesionales. El interés por la carrera y el desarrollo de estos equipos, unido al sentimiento de comunidad, al fenómeno fan que generan estos juegos, facilitan la creación de contenidos de marca, vinculados a ofrecer a los seguidores del equipo un valor añadido más allá de la participación o visionado de los deportes electrónicos.

Sin embargo, en opinión de los autores del presente artículo, no ha llegado el momento en el que los deportes electrónicos hayan recibido la atención global interdisciplinaria y menos aún han adquirido el reconocimiento suficiente como para poder sugerir siquiera que ya puede hablarse incluso del cientificismo en el e-sports. A pesar de ello, numerosos expertos y estudiosos del comportamiento social y humano, de los cuales se han recogido en el manuscrito algunas de sus impresiones, se han decantado por descubrir en estas dinámicas el perfil atlético en algunos gamers, lo cual brinda luces hacia un futuro cercano donde jugar con videojuegos deje de ser considerado pueril. Por su parte Roncero (2019), expone que:

Desde el 2006 investigadores de la talla de Brett Hutchins, estudian las competiciones profesionales de videojuegos, como un fenómeno a medio camino entre el deporte y el espectáculo mediático, analizando las relaciones de los e-sports con los medios tradicionales, las retransmisiones y su alcance social. Hutchins, continúa profundizando en esta línea y estudia las relaciones existentes entre el deporte, los e-sports y los medios de comunicación. El mencionado autor, plantea la necesidad de estudiar los deportes electrónicos como un fenómeno tecnológico-social, como reflejo de un cambio en las relaciones sociales y explora los vínculos entre los e-sports y el deporte tradicional a través de la figura del jugador profesional... al que identifica como cyberathletes.

Finalmente, los autores del presente artículo consideran fundamental exponer las ideas de otro experto en la temática, donde ciertamente palabras más ...palabras menos, se decanta por una postura similar a la que ellos comparten; donde sin pretender elevar y mucho menos infravalorar al videojuego, reconoce en el cierto valor por descubrir o redescubrir, como fenómeno humano, cultural, social, deportivo y moderno; el cual resultaría significativo para quienes como ellos han manifestado gran interés por los estudios del hombre y su pensamiento. En palabras de Roncero (2019), sucede que: 
Los e-sports no son todavía un objeto de estudio científico relevante, ni el término está aún extendido en la literatura académica, pero empiezan a surgir otros teóricos que ponen el foco de atención en este fenómeno. La mayoría de estas contribuciones se realizan desde una perspectiva social, que estudia el videojuego competitivo como parte de la cultura popular de la época.

\section{CONCLUSIONES}

La masificación del videojuego, es un fenómeno que en opinión de los autores tiene su origen en la naturaleza masculina, si bien es muy cierto que existen gamers del sexo femenino, es más cierto aún que todo este revuelo comenzó con el uso y aprovechamiento que los usuarios masculinos le dieron al videojuego desde su origen, por allá a principios de los 80 's... con juegos tan irónicos como el de Súper Mario Bross, por mencionar alguno, el cual tras casi cuatro décadas sigue siendo tendencia y ha recibido las mayores ovaciones desde su lanzamiento y todo ello tiene que ver con este tema de lo visual, los hombres son seres visuales, de allí que orienten su interés hacia lo colorido, lo estético, rutilante, multiforme y si además se le adiciona el factor dinámico - competitivo o incluso el elemento de búsqueda y cacería... naturalmente se iba a sentir atraído a intervenir en ello.

Con atención al párrafo anterior, debe tenerse en cuenta que con el surgimiento del internet, tuvo origen a una nueva dimensión a la recreación con videojuegos, pues ofreció oportunidades de diversa naturaleza, multiplicó su dinamismo al permitir que pudiera jugarse en tiempo real con otros usuarios... que inclusive pudieran estar en otras latitudes, indiferentemente del país en que se encuentren; lo cual fue aporte de otro fenómeno de interés múltiple como lo es la globalización, de esta se han desprendido otros eventos con ocasión a la masificación en la interacción y socialización entre personas sin importar su origen, y como agregado fundamental, se reconoce al impacto que diariamente ejercen sobre el comportamiento humano, el desarrollo y utilización de las TIC, en lo que no sólo se tiene en cuenta a los dispositivos electrónicos y digitales, también se hace hincapié en las plataformas de comunicación que robustecen al evento e-sports.

Sin embargo, como se explicó a lo largo del presente manuscrito, la evolución que en los últimos 40 años experimentaron los videojuegos, al dejar de ser una actividad destinada a la recreación y desarrollo lúdico infantojuvenil, transformándose en un evento de alcance internacional; con apoyo a los elementos expuestos en el párrafo anterior a este; favorece la potencialización del comportamiento humano en disímiles dimensiones: intrapersonal, interpersonal, lógico-espacial, racional, emocional, entre tantas que van de una persona a otra, según su personalidad, sexo, género, origen y nivel cultural. Los e-sports configuran la gamification del deporte, donde los gamers más destacados y reconocidos a nivel internacional e institucional, han alcanzado el estatus de cyberathletes... más no deportistas.

Ahora bien, en opinión de los autores de este artículo, resulta conveniente generar nuevas prácticas investigativas, donde quizás con la aplicación de otras técnicas... tal vez del tipo cualitativo, se logre auscultar el trasfondo social y cultural del videojuego, elevado a un estatus deportivo en la figura del e-sports, lo cual pudiera generar hallazgos con mayor profundidad, ante la vertiginosidad de los tiempos que corren y el dinámico comportamiento del hombre moderno. Finalmente, para concluir se quiere dejar por asentado que quienes generaron este extenso, están claros en que el mismo reviste un cuerpo escrito documental y 
reflexivo, lo cual no implica la exposición categórica de teorías conclusivas con referencia a la temática abordada; más sin embargo, configura la oportunidad en la que los expertos plantean laudos epistémicos, que dan origen al interés por nuevas y futuras investigaciones que den cuenta del evento referido.

\section{REFERENCES}

Aranda, D., Sánchez, J. y Martínez, S. (2016). El videojuego en el punto de mira: La producción científica sobre el juego digital. GAME. Recuperado de https://www.researchgate.net/publication/308916823

Arias, G. (2012). El proyecto de Investigación. Introducción a la metodología científica. Caracas. Venezuela. Editorial Episteme.

Beltrán, V., Valencia, A. y Molina, J. (2011). Los videojuegos activos y la salud de los jóvenes: revisión de la investigación. Revista Internacional de Medicina y Ciencias de la Actividad Física y del Deporte, 11(41), 203-219.

Checa, A. (2009). Hacia una industria española del videojuego. Revista Comunicación, 7(1), 177-188.

Chiavenato, I. (2009). Comportamiento organizacional. La dinámica del éxito en las organizaciones. Segunda edición. México. Editorial McGraw-Hil.

Chiva-Bartoll, O., Pallarès-Piquer, M. y Isidori, E. (2017). eSports y deportes convencionales: cuestiones éticas y pedagógicas derivadas de la participación corporal. Revista Calle Libre, 15(14), 71-79.

Fanjul, C., González, C. y Peña, P. (2019). La influencia de los jugadores de videojuegos online en las estrategias publicitarias de las marcas. Comparativa entre España y Corea. Revista Cientifica de Educomunicación, 58(27), 105-114. DOI: https://doi.org/10.3916/C58-2019-10

Fundación Telefónica (2017). eSports, de la pantalla a los estadios. Recuperado de http://fundaciontelefonica.com/

García, A. (2019). MAD Lions Esports Club: Experiencia profesional del psicólogo del deporte. Revista de Psicología Aplicada al Deporte y al Ejercicio Físico, 4(7), 1-11. DOI: https://doi.org/10.5093/rpadef2019a6

García, J. y Santizo, J. (2010). Análisis de la relación entre la gestión del tiempo libre, el ocio y los estilos de aprendizaje. Revista Estilos de Aprendizaje, 5(3), 2-25.

Gutierro, J. (2019). Entender al ser humano desde lo orgánico. La propuesta de Jonas y Plessner. Revista PENSAMIENTO, 75(283), 219-235.

Hernández, M. y Buitrago, R. (2017). Rol del sinestéta organizacional en el manejo de la comunicación asertiva. Económicas CUC, 38(2), 61-76. DOI: http://dx.doi.org/10.17981/econcuc.38.2.2017.05

Hernández, R., Fernández, C. y Baptista, P. (2006). Metodología de la investigación. México. Editorial McGrawHill.

Kopp, J. (2017). Juegos, juguetes y dispositivos tecnológicos. Revista Lúdicamente, 6(11), 1-17.

Lechuga, J., Leyva, O. y Cazallo, A. (2018). Liderazgo y desarrollo humano sostenible. En J. Lechuga, O., Leyva, A., Cazallo, U., Paz, A. Paz, N., Campo, ... G. Hennessey, R. Prieto, J., García, e Y., Rincón. (Comp.), Liderazgo: tendencias emergentes para inspirar la gestión de personas en las organizaciones (pp.7-30). Barranquilla: Ediciones Universidad Simón Bolívar.

Roncero, M. (2014). Deportes electrónicos. Una aproximación a las posibilidades comunicativas de un mercado emergente. Revista Questiones Publicitarias, 1(19), 98-115.

Roncero, M. (2019). Los deportes electrónicos (esports): el espectáculo de las competiciones de videojuegos. (Tesis doctoral publicada) Universidad Complutense de Madrid. Madrid. España.

Sabino, C. (2006). Los caminos de la ciencia: una introducción al método cientifico. Buenos Aires. Argentina. Editorial Lumen Humanitas.

Salcedo, M. (2014). Videojuegos: realidad que desborda los límites. Acercamiento teórico de posibles modificaciones cognitivas y funcionales en jóvenes. Revista de Investigación: Cuerpo, Cultura y Movimiento, 4(2), 163-170.

Sánchez, M., Ramirez, I., Baez, F., Moreno, R. y Fernández, A. (2019). Análisis de la relación existente entre el uso de videojuegos y la práctica de actividad física. Sportis Sci J, 5(1), 118-132. DOI: https://doi.org/10.17979/sportis.2019.5.1.3433

Santos, A., Alloza. S. y Escribano, F. (2018, 11 de abril). Manual para educadores: Relación entre géneros de videojuegos y soft skills. +1! Level Up. Recuperado de: http://gecon.es/game-genders-and-soft-skills

Sierra, R. (2010). El método investigativo. Caracas. Venezuela. Editorial Suramérica.

Tamayo y Tamayo, M. (2007). El proceso de investigación científica. México. Editorial Prentice Hall. 